\title{
UCRL-CONF-217045
}

LAW RENCE LIVERMORE N A T IO N A L LABORATORY

\section{Radiation Imaging of Dry-Storage Casks for Spent Nuclear Fuel}

K.-P. Ziock, G. Caffrey, A. Lebrun, L. Forman, P. Vanier, J. Wharton

November 11, 2005

2005 IEEE Nuclear Science Symposium and Medical Imaging Conference

Fajardo, PR, United States

October 23, 2005 through October 29, 2005 
This document was prepared as an account of work sponsored by an agency of the United States Government. Neither the United States Government nor the University of California nor any of their employees, makes any warranty, express or implied, or assumes any legal liability or responsibility for the accuracy, completeness, or usefulness of any information, apparatus, product, or process disclosed, or represents that its use would not infringe privately owned rights. Reference herein to any specific commercial product, process, or service by trade name, trademark, manufacturer, or otherwise, does not necessarily constitute or imply its endorsement, recommendation, or favoring by the United States Government or the University of California. The views and opinions of authors expressed herein do not necessarily state or reflect those of the United States Government or the University of California, and shall not be used for advertising or product endorsement purposes. 


\title{
Radiation Imaging of Dry-Storage Casks for Spent Nuclear Fuel
}

\author{
Klaus-Peter Ziock, Gus Caffrey, Alain Lebrun, Leon Forman, Peter Vanier and Jason Wharton
}

\begin{abstract}
We report the results of a measurement campaign conducted on six dry-storage, spent-nuclear-fuel storage casks at the Idaho National Laboratory. A gamma-ray imager, a thermalneutron imager and a Ge-spectrometer were used to collect data. The campaign was conducted to examine the feasibility of using cask radiation signatures as unique identifiers for individual casks as part of a safeguards regime. The results clearly show different morphologies for the various cask types although the signatures are deemed insufficient to uniquely identify individual casks of the same type. Based on results with the Gespectrometer and differences between thermal neutron images and neutron-dose meters, this result is attributed to the limitations of the extant imagers used, rather than of the basic concept.
\end{abstract}

\section{INTRODUCTION}

A ccounting for spent nuclear fuel is one of the important safeguarding tasks performed by the IAEA. The material is both highly radioactive and can contain significant quantities of fissile material. For the ten years following the last burn cycle, the material is generally stored in wet-storage facilities or "ponds" at the reactor site. After this time, it can be transferred to dry storage casks and moved to pads awaiting final disposal. Despite the fact that we are over five decades into the nuclear era, no long-term disposal sites exist. This means that more and more material is accumulating at "temporary" storage facilities in dry storage casks.

Although there are many types of dry storage casks, they all share the basic design goal to limit radiation exposure outside the cask. This means that cask walls include significant shielding for both neutron and gamma-radiation. Hence, traditional non-destructive analysis (NDA) and assay techniques to verify cask contents are ineffective after a cask is sealed. The safeguard regime therefore relies on the use of tamperproof tags and seals $(\mathrm{C} / \mathrm{S})$ with regular inspections to maintain continuity of knowledge that cask contents remain as loaded. Loading occurs in the presence of IAEA inspectors who apply the $\mathrm{C} / \mathrm{S}$ indicators after the cask is "irreversibly" sealed. The casks are generally stored in "farms" that are periodically visited by inspectors who certify that the cask contents have not been altered.

Manuscript received November 11, 2005

K. P. Ziock was with Lawrence Livermore National Laboratory, Livermore, CA 94550 USA, he is now with Oak Ridge National Laboratory, Oak Ridge, TN 37831 USA (corresponding author e-mail: ziockk@ornl.gov).

G. Caffrey and J. Wharton are with Idaho National Laboratory, Idaho Falls, ID 83415 , USA.

L. Forman and P. Vanier are with Brookhaven National Laboratory, Upton, NY 11973 USA

A. Lebrun is with the International Atomic Energy Agency, Vienna Austria.
As the inventory of spent fuel around the world increases, monitoring of cask loading operations and subsequent $\mathrm{C} / \mathrm{S}$ inspections are creating a greater burden on IAEA resources. The ever-increasing quantity of casks also increases the likelihood of $\mathrm{C} / \mathrm{S}$ failure. This is particularly problematic since farms are generally outdoors, exposed to the weather. In the event of $\mathrm{C} / \mathrm{S}$ failure, there is currently no means of reestablishing a cask's contents without reopening it - a technique that is not considered realistic in light of the costs associated with such an undertaking.

In the late winter of 2003, the IAEA held a Coordinated Technical Meeting on Spent Fuel Verification Methods to examine the means of safeguarding spent nuclear fuel. One of the suggestions from the meeting was to use "fingerprinting" as a means to re-certify the contents of a cask in the event of $\mathrm{C} / \mathrm{S}$ failure and also as a means of strengthening the $\mathrm{C} / \mathrm{S}$ regime itself. The general concept relies on identifying properties unique to individual casks, based on their loading, that can be used to identify them independent of external markings.

At the heart of the technique are the facts that the casks are loaded with fuel assemblies of varying burn-up to distribute the heat load in the cask and that radiation from the fuel reaches the cask surface despite the heavy shielding. In principle, this should result in a multi-lobe signature of the radiation field that might serve to identify the cask loading.

The radiation pattern will have two components. The first, and major, component will be due to radiation that interacts in the cask walls on the way to the surface. This radiation will generate a diffuse image, such as that obtained from a light bulb behind a translucent diffuser. The second, smaller component will exit the cask without interacting in the shielding at all. This radiation might provide a faithful picture of the geometry of the outer nuclear materials. (The inner fuel elements will be shielded by the outer layers and will be difficult to image directly.)

Based on this concept we conducted a measurement campaign including both a thermal-neutron and a gamma-ray imager at the Test Area North (TAN) site of the Idaho National Laboratory (INL). This facility has six different spent fuel casks available for test measurements (See Fig. 1.)

\section{INSTRUMENTATION}

\section{A. Coded aperture imaging}

The two imagers used in the measurements are based on the coded-aperture imaging technique.[1] This form of imaging relies on a shadow mask placed between the radiation source 


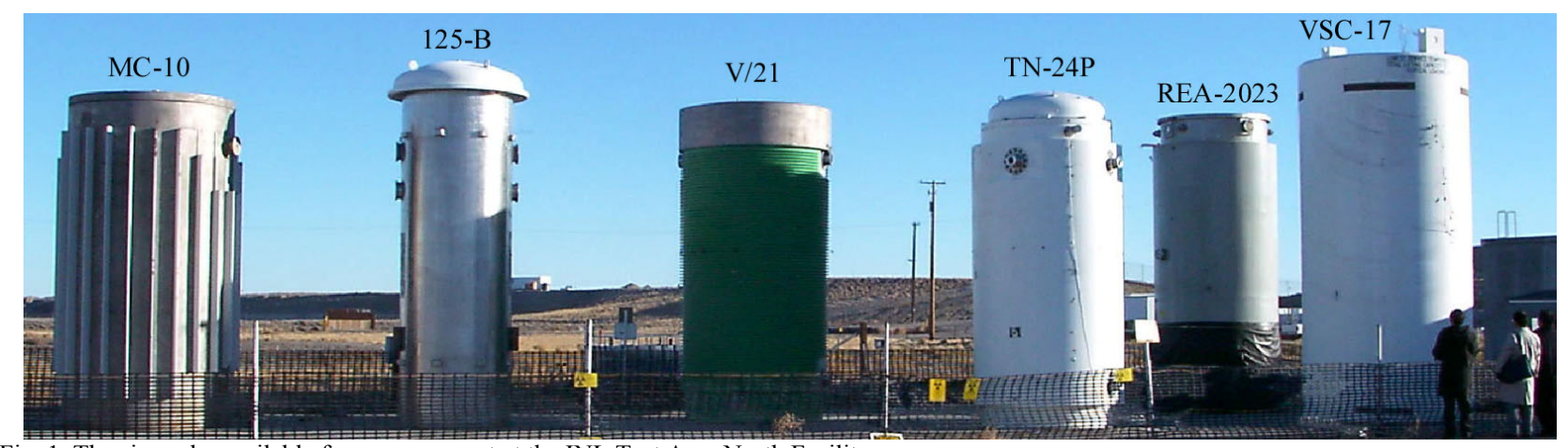

Fig. 1. The six casks available for measurement at the INL Test Area North Facility.

and a position-sensitive detector to encode the image as a spatial modulation of counts at the detector. With specially selected shadow patterns, artifact-free images can be obtained in times short compared with those required by a pinhole camera. To remove artifacts due to various effects, the data is normally collected in two equal-time integrations using a mask and its inverse (anti-mask.) The shadow patterns used by both of the imagers are anti-symmetric on rotation and this property is used to obtain the two data sets.

\section{B. Gamma-ray Imager}

The gamma-ray imager used in these measurements is shown in Fig. 2. It is described in detail elsewhere. [2] It comprises a base-19, HURA, coded-aperture mask [3] that is projected onto a $\mathrm{CsI}(\mathrm{Na})$-based, position-sensitive, gamma-ray detector. The mask is $5-\mathrm{mm}$ thick Ta with a hexagonal hole flat-face size of $2.14 \mathrm{~mm}$. The detector uses a $12-\mathrm{cm}$ diameter, 1-cm thick CsI(Na) disk mounted on a Hamamatsu R3292 [4] position-sensitive-photomultiplier tube (PSPMT). The detector has an effective area of $\sim 60 \mathrm{~cm}^{2}$, a position resolution of 3 $\mathrm{mm}$ and an energy resolution of $11 \%$ at $356 \mathrm{keV}$. The detector is mounted on linear bearings that allow it to be set in one of five different distances from the mask, providing a zoom capability. Data from the PSPMT is collected by a gatedintegrator-based data acquisition system that uses a commercial, 16-bit digitizer mounted in a palm-top computer. The

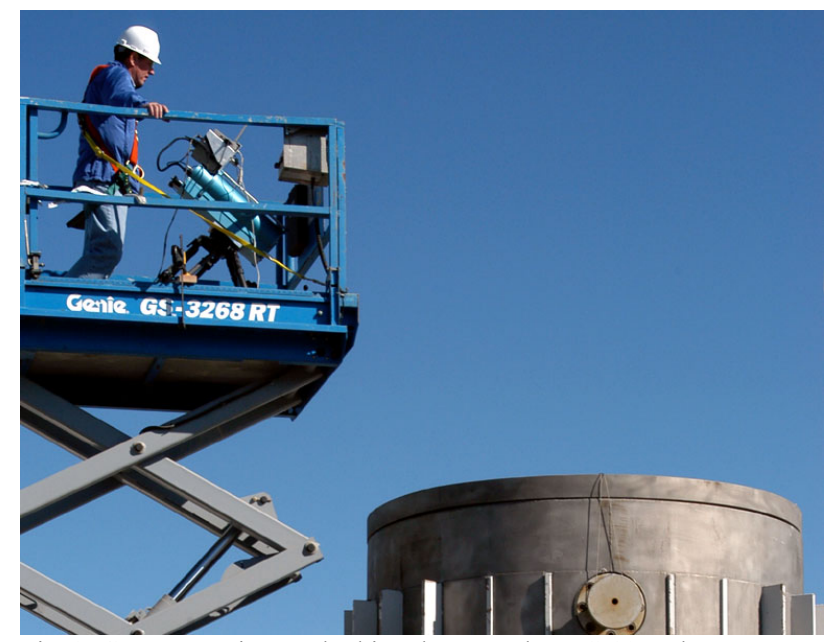

Fig. 2. Gamma-ray imager looking down on the MC-10 cask. computer records the data in list-mode and also generates an online image.

In addition to the gamma-ray data, a visible-light video camera, coaligned with the imaging axis of the instrument, is used to record an image before each run. This image is scaled to the gamma-ray image and the two are simultaneously displayed in an overlay mode by the computer. The gamma-ray image is shown in false-color "in front" of the visible light image. Those gamma-ray pixels a selectable value below the maximum are turned clear so that portions of the visible light image can be seen.

The list-mode data file recorded during an acquisition can be used to perform a complete, post-acquisition analysis, allowing the user to vary software settings (such as source distance and energy cuts.) The data can also be processed to create images for each energy bin, generating a data cube that can be explored interactively to determine both the images obtained in different energy windows, and the spectra from different pixels or regions of the image. [5]

Finally, it should be noted that the gamma-ray imager used in these measurements is a prototype developed for use in arms-control applications. As such, it is optimized to respond to gamma-radiation from ${ }^{235} \mathrm{U}$ at $186 \mathrm{keV}$ and the 374 and 414 $\mathrm{keV}$ lines from $\mathrm{Pu}$ as well as the $\sim 100 \mathrm{keV}$ K-shell fluorescence radiation from these elements. Unfortunately, the upper limit of the electronics is set to $630 \mathrm{keV}$, a value that is too low to image any of the line radiation that was measured to leak from the casks with the Ge spectrometer.

\section{Thermal Neutron Imager}

The thermal neutron imager is based on a crossed-wire chamber with a $20-\mathrm{cm} \times 17-\mathrm{cm}$ sensitive area operated in the proportional mode with a gas mixture of 6 bar $^{3} \mathrm{He}$ and $2.5 \mathrm{bar}$ of propane. Details of the construction and the readout electronics can be found elsewhere [6]. The detector is enclosed in a Cd-lined box equipped with one of three choices of codedaperture, MURA masks [7] fabricated from 0.4-mm thick Cd mounted on an unperforated aluminum backing sheet. A mask can be inserted in one of four different tracks at different focal lengths. The base pattern of each mask is a square of $15 \mathrm{~cm} \mathrm{x}$ $15 \mathrm{~cm}$. Three different resolution masks are available with (A) $19 \times 19$ (B) $31 \times 31$ and (C) $47 \times 47$ pixels in the base pattern. The majority of cask data was collected with a $10-\mathrm{cm}$ 


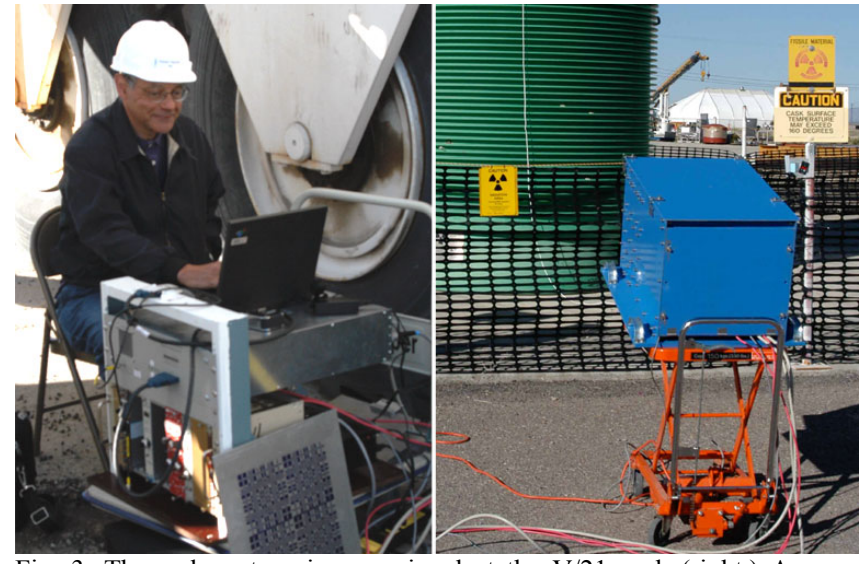

Fig. 3. Thermal neutron imager aimed at the $\mathrm{V} / 21$ cask (right.) A spare mask and the remote data acquisition system (left.)

focal length. This gives a field of view at the source that is 1.5 times the distance to the cask. The imager is shown in Fig. 3.

A custom-built, data-acquisition board is used to accumulate a 2-d spatial histogram of neutron absorption events. The spatial resolution of the chamber is limited to about $1 \mathrm{~mm}$ by the difference in ranges of the proton and triton produced by the reaction of a neutron with ${ }^{3} \mathrm{He}$. This is oversampled threefold by the readout electronics. This means that the smallest mask pixel $(3 \mathrm{~mm} \times 3 \mathrm{~mm})$ is oversampled by about a factor of 9 in each direction, providing a high degree of adjustability in the image-processing software. We use this to allocate regions of the detector to pixels of the mask shadow while taking account of the magnification of the shadow at short ranges. [5] Optimizing the focus for objects in the scene at different ranges can therefore be performed in software after the data are recorded. Also, physical registration of detector elements with mask pixels is not required.

\section{Gamma-ray Spectrometer}

The high-resolution, gamma-ray spectra from the casks were taken using a $39 \%$ relative-efficiency, N-type, coaxial, high-purity germanium detector. It is collimated with a bismuth annulus restricting its field of view to 10 degrees (Fig. 4.) The system is the same as used for PINS work and has been described in detail elsewhere [8]. The data were collected using an ORTEC, Nomad-Plus, portable, multi-channel analyzer. The deadtime of the measurements was kept below $26 \%$. Data were collected from a distance of $3 \mathrm{~m}$ and the de-

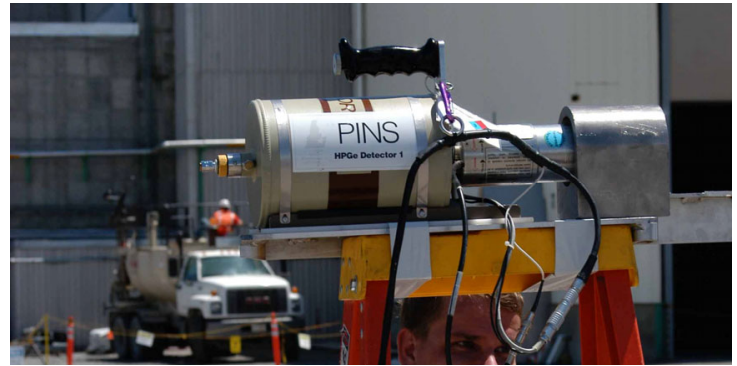

Fig. 4. The Ge spectrometer and collimator.

tector was elevated $1.8 \mathrm{~m}$ above the ground, nearly at cask mid-height.

\section{TESt AREA North (TAN) FACILITY}

The "Test Area North" at Idaho National Laboratory (INL) has a facility to perform experimental measurements on six different, spent-nuclear-fuel, dry-storage casks. These are set outdoors on a concrete pad with a fence providing a personnel boundary between the general and a radiation zone. Flux at the boundary can be as high as $5 \mathrm{mR} / \mathrm{hr}$. All images save the overhead views were taken from outside the fence. The casks with images in the paper and a synopsis of their loading, is given below, details are available from [9]:

1) Westinghouse $M C$-10: This cask contains 5 intact Turkey Point PWR assemblies, 12 VEPCO PWR assemblies and one BCD B-17 PWR assembly. The latter has 21 fuel rods replaced by stainless steel rods for a total of 183 fuel rods. Thus 18 of 24 possible locations are used for a total of 14.99 Metric Tons of Heavy Metal (MTHM).

2) GNS Castor V/21 Cask: This Cask contains 21 VEPCO PWR assemblies. Twenty of the assemblies are intact while one has 12 rods removed for a total of 9.27 MTHM. The fuel was discharged from the Surry reactor in Nov. 1981 and was in water storage until July 1985 when it was loaded into the cask. [10] On average it has a burn-up of 30-35 GWd/MTU. [11]

3) REA-2023: This cask is loaded with intact commercial fuel assemblies from Connecticut Yankee (PWR,) H. B. Robinson (PWR,) Beach Bottom (BWR) and Dresden I (BWR) reactors. Five positions in the cask are occupied by "loose" fuel rods. The cask also contains fuel from the Loss of Fluid Test (LOFT) reactor including the center module FP-1 with two rods removed and the remains of
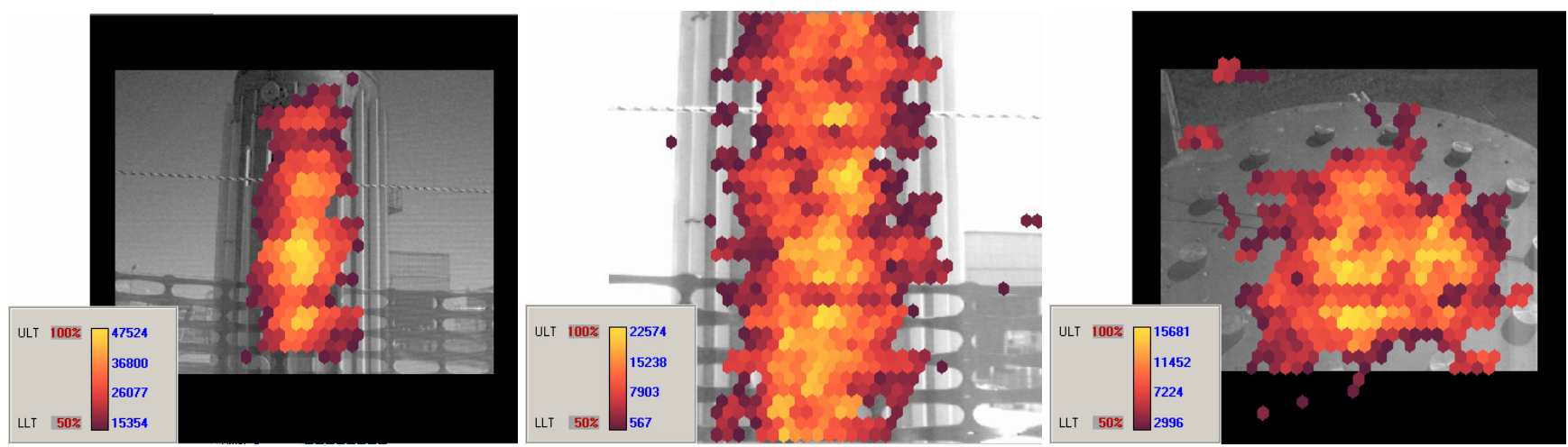

Fig. 5. Gamma-ray images of the MC-10 cask. The wide-angle image (left) and zoomed-image (middle) show little structural detail but are clearly of a different shape than the top view (right.) The spectrum from the middle image is shown in Fig. 6. 


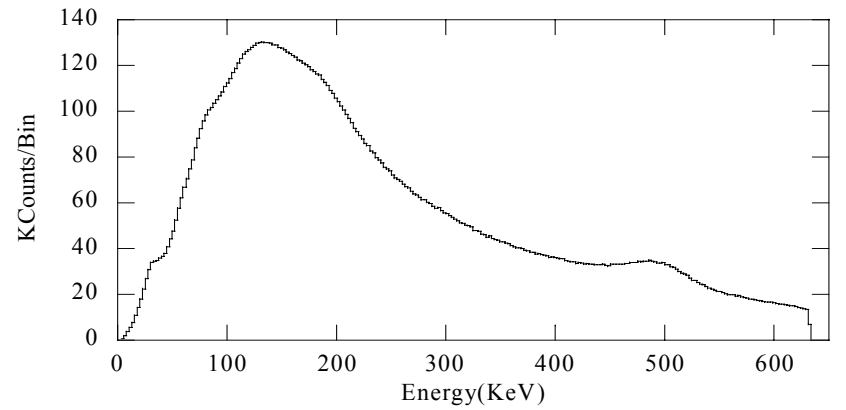

Fig. 6. Spectrum from the zoomed, side-view image of the MC10 cask.

the FP-2 center module housed in ten storage cans that also contain two loose FP-rods and epoxy. The total Cask content is $1.79 \mathrm{MTHW}$

\section{MEASURMENTS}

\section{A. Westinghouse $M C-10$}

The most extensive measurements were performed on the Westinghouse MC-10 cask. Its location at the end of the pad allowed images to be obtained without concerns that radiation from neighboring casks also cast shadow patterns on the detectors, possibly resulting in artifacts.

\section{1) Gamma-ray imager data}

Gamma-ray images from this cask are shown in Fig. 5. Two side views of different resolutions were obtained from a range of $6.14 \mathrm{~m}$. The wide-field image represents $120 \mathrm{~min}$. of data with pixels at the cask $14 \mathrm{~cm}$ across. The higher-resolution image has pixels $8.5 \mathrm{~cm}$ across and represents $180 \mathrm{~min}$. of data. Finally, a lift was used to obtain an oblique, top-down, view. This image represents a total 160 mins. of data at a spatial resolution of $\sim 7.0 \mathrm{~cm}$. A sample spectrum obtained with the imager is shown in Fig. 6. Although there are some differences between the end and side spectra, the differences are not worth exploring given the low resolution of the device.

\section{2) Thermal neutron imager data}

A side view of the cask was obtained $8.1 \mathrm{~m}$ from the center of the cask with a $10-\mathrm{cm}$ focal length, using the " $\mathrm{A}$ " mask. The field of view was 12.2 meters, with $64-\mathrm{cm}$ pixels at the cask. The count rate was about 250 neutrons per second. The image comprises six hours of data. Fig. 7 shows the final im-
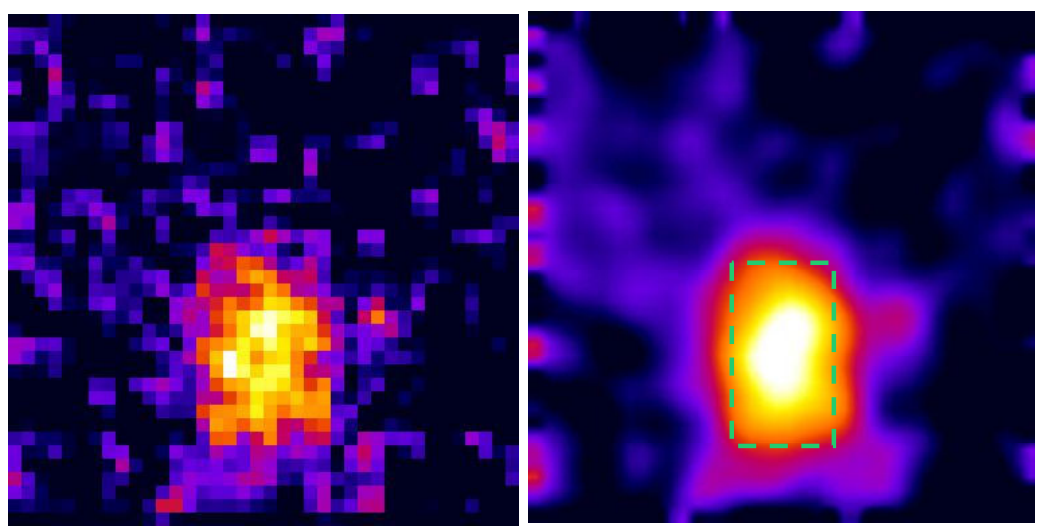

Fig. 7. Thermal neutron images of the MC-10 cask, normal (left) and smoothed (right.) The dashed rectangle shows the outline of the cask.

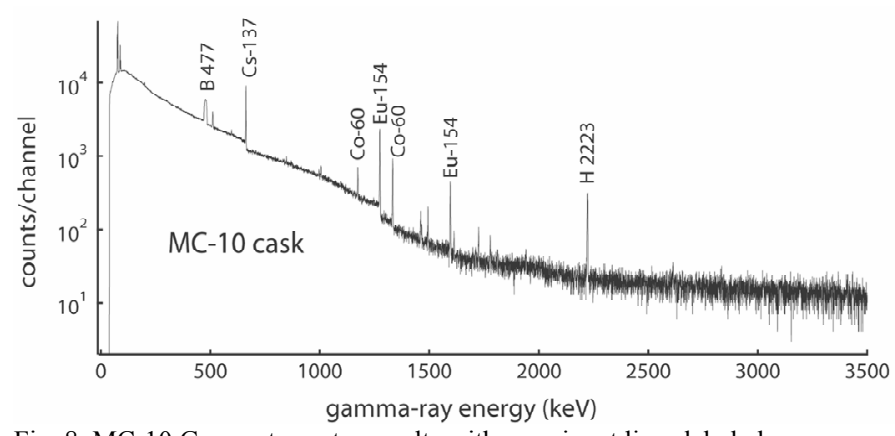

Fig. 8. MC-10 Ge spectrometer results with prominent lines labeled.

age with unsmoothed data binned into areas corresponding to $1 / 4$ of the mask pixel area. It is not clear whether fluctuations in intensity on the scale of one pixel show any significant features in the fuel assembly, because the image still contains a lot of statistical noise. A smoothed image is also shown with the outline of the cask superimposed in green. It can be seen that the entire cask glows fairly uniformly. Top-down views similar to those obtained with the gamma-ray imager were attempted, but no useful image resulted.

3) Ge Spectrometer data

A spectrum obtained on this cask with the Ge detector is shown in Fig. 8. The data represent $1000 \mathrm{sec}$. of livetime with a deadtime fraction of $22.9 \%$. The spectrum shows prominent gamma-ray lines of the fission products ${ }^{137} \mathrm{Cs},{ }^{60} \mathrm{Co}$ and ${ }^{154} \mathrm{Eu}$; and contains boron, calcium, hydrogen, iron and silicon neutron induced gamma-ray peaks as well.

\section{B. Other Casks}

Spatial constraints restrict displaying all of the images obtained during the campaign. Sample images demonstrating different morphologies seen are shown in Fig. 9 for both gamma and neutron images. The images on the left are of the Castor V/21 cask and those on the right of the REA-2023. The gamma-ray threshold of the left image is $60 \%$ due to poor contrast. All other gamma images have a threshold of $50 \%$.

\section{DISCUSSION}

As can be seen by examining the various images, the results clearly differ by both cask type and the radiation that is imaged. The most striking example of the latter is shown on the left side of Fig. 9 where the gamma-ray and thermal neutron images of the Castor $V / 21$ are presented. The gamma-ray image has low-contrast with the prominent feature a hot region at the bottom of the cask. This is likely due to radiation emitted from the poorly shielded cask bottom scattering into the imager's line of sight. In contrast, the thermal neutron image shows the cask as a region of low emission against a brighter background. This is particularly surprising given that this cask showed one of the highest neutron doserates as measured by the health physicists. This can be explained by the fact that dose measurements are taken with detectors that include thermalizing material, i.e. poly-ball detectors. This is 


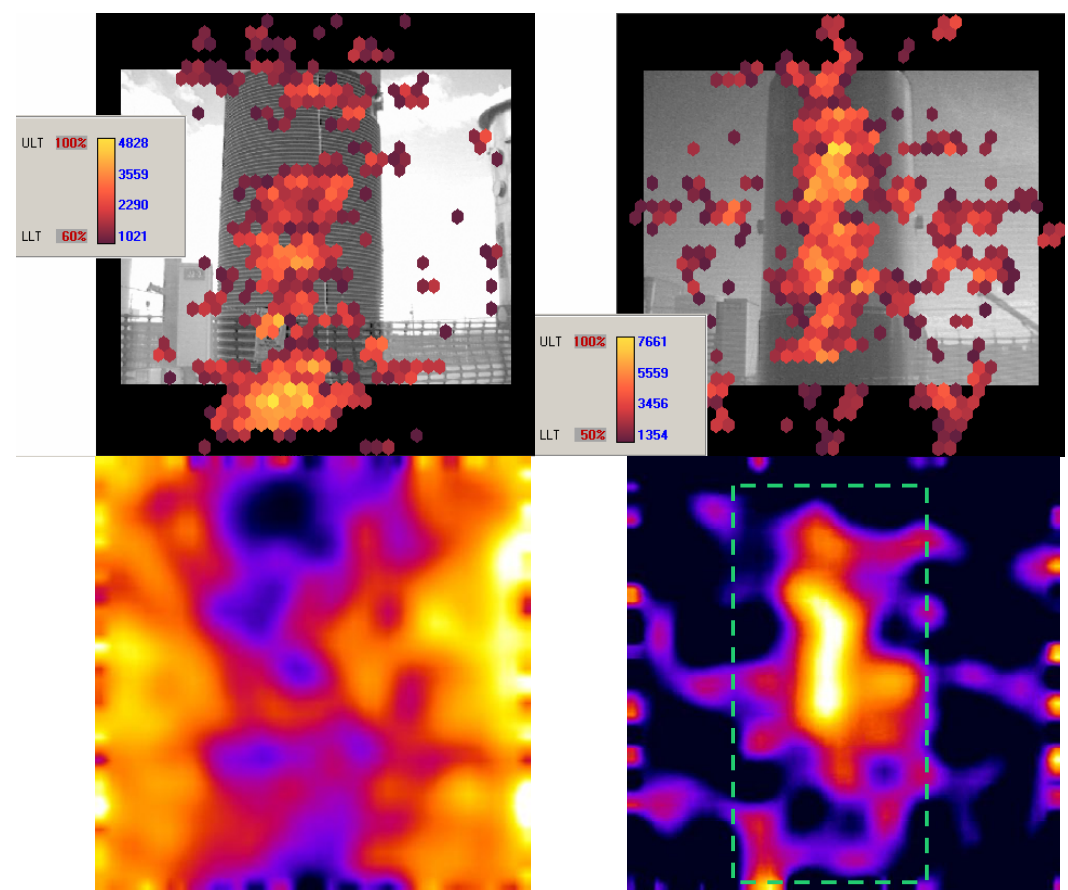

Fig. 9. Sample images of the Castor V/21 cask (left) and the REA-2023 cask (right). The gamma-ray images are shown in the top row with the corresponding thermal neutron images shown in the bottom. The bottom left neutron image reveals the cask as a region of low flux.

\section{CONCLUSION}

Although different cask types can be identified using radiation imaging, the images obtained with thermal neutron and low energy gamma-ray imagers are dominated by radiation that is scattered in the shielding. This scattered component overwhelms any residual unscattered radiation and its contribution to the image. This indicates that cask fingerprinting is not possible with such instruments. However, evidence that significant fluences of higher-energy, unscattered radiation exit the casks exists. Images obtained with instruments sensitive to this radiation may provide the requisite clarity to allow fingerprinting to succeed. Such devices are currently under development.

\section{ACKNOWLEDGEMENT}

This work was performed under the auspices of the U.S. Department of Energy by University of California, Lawrence Livermore National Laboratory under Con-tract W-7405-Eng-48.

\section{REFERENCES} thermal energy range that the imager sees.

By comparison the REA 2023 cask on the right side of the figure shows similar morphologies with both radiation types. The radiation is well contained to the middle of the cask.

In general the results clearly indicate that the images obtained are dominated by the radiation component scattered in the shielding. This explains why the gamma-ray images show little structural detail - the diffuser in front of the light source is too thick. The thermal neutron images are one step worse, directly correlating with the type of shielding material. Those casks with concrete shielding show a well defined thermal image, while those without do not.

Despite these disappointing results, there is some hope that fingerprinting techniques may be viable. The Ge spectra, as represented by Fig. 8, show significant promise. In particular, the prominent photopeak lines in the spectra must come from unscattered radiation, since Compton scattered gamma-rays show up below the photopeak energy. Hence, gamma-rays in the photopeak did not scatter in the shielding. Further, the signal-to-background ratios in the lines are of order unity. This would indicate that if one had an imager suitable for use at higher energies (say the ${ }^{137} \mathrm{Cs} 662 \mathrm{keV}$ line) and with good energy resolution, then one could obtain clearer images. Such imagers based on Ge-strip detectors using either coded aperture or Compton techniques are under development. [12, 13]

Even the neutron picture is not without promise. The fact that higher energy neutrons are emitted by at least one cask means some of this radiation may emerge with little or no scattering as well. Hence, fission-spectrum-neutron imagers may be suitable for this application. Such instruments based on scattering techniques similar to gamma-ray Compton cameras are also currently under development. [14]
[1] E.E. Fenimore, T.M. Cannon, "Coded aperture imaging with uniformly redundant arrays," App. Opt., vol. 17, no. 3, pp. 337-347, Feb. 1978.

[2] K.P. Ziock, L. Nakae,." A Large-Area PSPMT Based Gamma-ray Imager with Edge Reclamation," IEEE Trans. Nucl. Sci., vol. 49, no. 3, pp. 1552-1559, June 2002.

[3] W. R. Cook, M. Finger, T.A. Prince, E.C. Stone, IEEE Trans. Nucl. Sci., vol. 31, pp. 771-775, Feb. 1984.

[4] Hamamatsu Photonics K.K., Toyooka-Village, Iwata-gun, Shizuoka-ken 438-0193, Japan.

[5] K.P. Ziock, M.T. Burks, W. Craig, L. Fabris, E.L. Hull, N.W. Madden, Real-time generation of images with pixel-by-pixel spectra for a coded aperture imager with high spectral resolution, NIM, vol. A505, no. 1-2, pp. 420-424, June 2003.

[6] P.E.Vanier, L. Forman, "Forming images with thermal neutrons," Proc. SPIE, vol. 4784A, pp. 183-193, 2002.

[7] S. Gottesman, E. Fenimore, "New family of binary arrays for coded aperture imaging," App. Opt. vol 28, 20, pp. 4344-4352, Oct. 1989.

[8] A.J. Caffrey, J.D. Cole, R.J. Gehrke, R.C. Greenwood, "Chemical Warfare Agent and High Explosive Identificiation by Spectroscopy of Neutron-Induced Gamma Rays," IEEE Trans. Nucl. Sci., vol 39, no. 5, pp. 1422-1426, Oct. 199.

[9] "INEEL Spent Nuclear Fuel Integrated Transfer Schedule," available from: http://www.id.doe.gov/DOEID/RFPSharedLibrary/refdoc.htm as: http://www.id.doe.gov/DOEID/RFPSharedLibrary/PDF/PLN\%20845\%20Rev\%202\%20from\%20DOC.pdf

[10] R.E. Einziger, H.C. Tsai, M. C. Billone, , B.A. Hamilton, Proc. of ICONE10, 10th Int. Conf. on Nucl. Eng., Arlington, VA, April 14-18, 2002.

[11] Transactions, SMiRT 16 paper \#1309, Washington DC, Aug. 2001.

[12] K.P. Ziock, N. Madden, E. Hull, W. Craig, T. Lavietes, C. Cork, IEEE Trans. Nucl. Sci. vol. 49, no. 4, pp. 1552-1559, Aug. 2002.

[13] K. Vetter, M. Burks, L Mihailescu, "Gamma-ray imaging with positionsensitive HPGe detectors, NIM, vol. A525, no. 1-2, pp.322-327, June 2004.

[14] P. Vanier, L. Forman, "An 8-element neutron double-scatter directional detector," to appear in Proc. SPIE, vol 5923, 2005. 